\title{
Reflective properties of Anti-forgery Optically variable films series
}

\author{
Xu Yue, Liu Fu-ping, Hao Yan-nan, Wang An-ling \\ (Beijing Institute of Graphic Communication, Beijing 102600,China) \\ afupingliu60@sina.com (corresponding author), ,wanganling@bigc.edu.cn
}

\begin{abstract}
Key words: Multi-layer anti-counterfeiting optical films; Vertical polarized wave; Reflection property
\end{abstract}

Abstract: For the optically variable films series, using the boundary conditions of electromagnetic waves, we have given the propagation wave equation of S-polarized light wave in the optical variable film system with razor-thin metal film, which can be used to calculate electromagnetic field distribution and the reflection coefficient for the light of any incident direction and the arbitrarily layered security film system. Based on the MATLAB programming we calculate the energy reflectivity of light for the security film system, we also analysis the reflective properties of the optical film system with some cases.

\section{Introduction}

Because of color ink print have dynamic color effect and a good hiding, when the viewing angle changes we can clearly see the changes of the color of the original pattern ${ }^{[1-2]}$. It has the good intuitive which eye can identify ${ }^{[3-5]}$, difficult to counterfeit. So color ink security printing technologies have important applications in bank notes, checks, bonds, stocks and other securities that require high security. To improve the narrowband high reaction of optical film, in the current a thin metal film is used with a dielectric multilayer film consisting of a combination of film system ${ }^{[1-2]}$, in such a membrane system containing a highly conductive metal thin film, however, since the metal film and the dielectric film conductivity have huge difference ${ }^{[7]}$ (in the order of $10^{10}$ ), therefore, the metal film to have light propagation effects in the membrane system is still very large. Considered the characteristics of thin metal films, in this paper, we adapt a simplified approach to the metal film and give a propagation equation of light wave in the multilayer film (metal film) for the non-normal incidence light, through the case we have analyzed the reflective properties of anti-forgery dielectric optically variable films series

\section{The Reflection Model Of Anti-forgery Optically Variable Films Series}

In Figure 1.two coherent beams 1 and 2 respectively reflect on the surface of the top and bottom of the layer, its difference in optical path is $\Delta=2 n d \cos i^{\prime}=2 d \sqrt{n^{2}-n_{1}^{2} \times \sin ^{2} i}$, The phase difference of the two beams can be written as $\delta=\frac{2 \pi}{\lambda} n d \cos i$. When the film is a multilayer optical variable film system, the matrix equation is obtained ${ }^{[8]}$ 


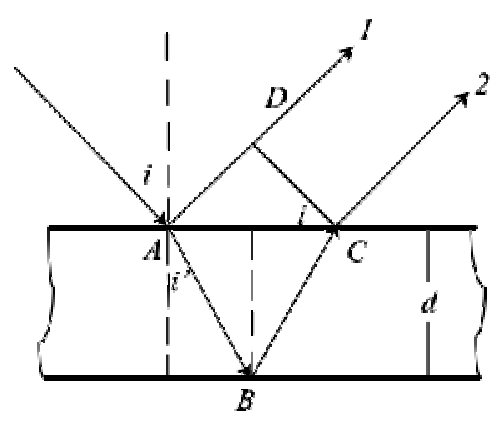

Fig1.Dielectric film interference schematic

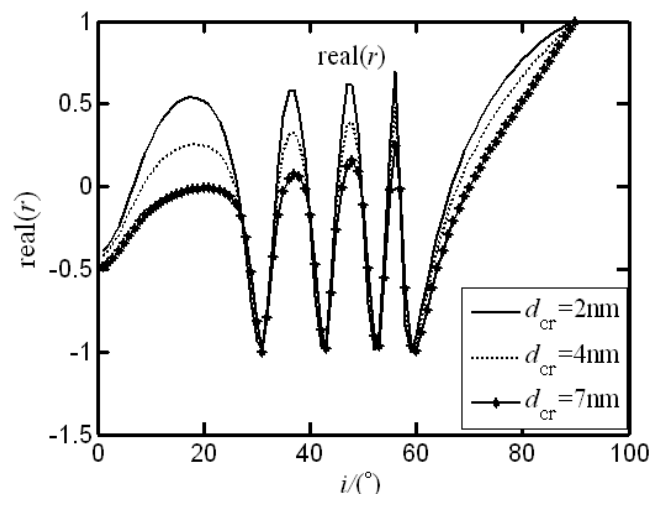

Fig 2 The reflection coefficient(real

part) of light of multilayer film

$$
\left[\begin{array}{l}
E_{1} \\
H_{1}
\end{array}\right]=\left\{\prod_{\mathrm{k}=1}^{K}\left[\begin{array}{cc}
\cos \delta_{k} & \frac{j}{\eta_{k}} \sin \delta_{k} \\
j \eta_{k} \sin \delta_{k} & \cos \boldsymbol{\delta}_{k}
\end{array}\right]\right\}\left\{\begin{array}{l}
E_{K+1} \\
H_{K+1}
\end{array}\right]
$$

Also because, $Y=H_{1} / E_{1}$, and on the substrate only positive wave, so $H_{K+1} / E_{K+1}=\eta_{K-1}$, we can have features matrix of multilayer optically variable film

$$
\left[\begin{array}{c}
E \\
H
\end{array}\right]=\left\{\prod_{\mathrm{k}=1}^{K}\left[\begin{array}{cc}
\cos \delta_{k} & \frac{j}{\eta_{k}} \sin \delta_{k} \\
j \eta_{k} \sin \delta_{k} & \cos \delta_{k}
\end{array}\right]\right\}\left\{\begin{array}{c}
1 \\
\eta_{K+1}
\end{array}\right]
$$

Where $\eta_{k}=n_{k} / \cos i_{k}$, because $Y=\frac{F}{E}$, thus the amplitude of the reflection coefficient can be obtained as follows $r=\frac{\eta_{1} E-F}{\eta_{1} E+F}$, Reflectance of the total energy $R=|r|^{2}$,

Since the geometrical thickness of the ultra thin metal film is very small, it can be simplified in calculation, feature matrix can be abbreviated as

$$
m=\left[\begin{array}{cc}
\cos \delta & \frac{j}{\eta} \sin \delta \\
j \eta \sin \delta & \cos \delta
\end{array}\right] \approx\left[\begin{array}{cc}
1 & j \frac{2 \pi}{\lambda} d \\
\frac{2 \pi}{\lambda} n k d & 1
\end{array}\right]
$$

where, complex refractive index of metal film $n^{*}=n-j \kappa$

\section{Computational example and its analysis}

The following calculation method of the study by a seven-layer asymmetric membrane system examples, membrane system for $\mathrm{A} / y \mathrm{M} 0.93 \mathrm{~L}(\mathrm{LH})^{2}(\mathrm{LH})^{2} 0.76 \mathrm{~L} 100 \mathrm{~N} / \mathrm{G}, \mathrm{A}$ medium is one of the most top, $\mathrm{G}$ is the lowest medium, $\mathrm{M}$ represents a chromium film, $\mathrm{N}$ denotes the silver film, $\mathrm{L}$ is a low refractive index dielectric film, $\mathrm{H}$ indicates a high refractive index dielectric film, Letter in 
front of the number indicates the thickness of the dielectric film (a film-based center wavelength multiples). In the membrane system, $n_{H}=2.35, n_{L}=1.32, P=2, n_{g}=1.52, n_{A}=1.52$, Chromium film thickness is $\mathrm{y}=2,4,7 \mathrm{~nm} . \mathrm{x}, \mathrm{z}$ for the L layer introduces phase compensation factor(Literature[1] adopt $x=0.93, z=0.76$ ), the film thickness of $\mathrm{Ag}$ is $100 \mathrm{~nm}$, center wavelength is $520 \mathrm{~nm}$, the conductivity of chromium film is $\sigma=7.752 \times 10^{6}$, the conductivity of the silver film is $\sigma=6.06 \times 10^{7}$, complex refractive indexes of Chromium and Silver film $n_{c r}=2.91-3.33 \mathrm{i}$ and $n_{a g}=0.13-3.07 \mathrm{i}$.From the figure 2 we can see that the amplitude of the reflection coefficient is decreasing with the increasing film thickness of metallic chromium, which is actually due to the increased absorption capacity of the chromium-based film is enhanced due to the film thickness.

From Figure 3 can also be found that the size of imaginary part of the reflection coefficient curve is decreasing with the chromium film thickness increasing, which is due to the presence of the metal film, the size of the imaginary part of the reflection coefficient curves accurately reflect the absorption effect of the metal film.

Figure 4 is the curves of the energy reflectance, where the main wavelength $\lambda=520 \mathrm{~nm}$. Figure 5 indicates that the total energy reflectivity thereof is gradually reduced with the film thickness of chromium increasing, which reflects the absorbing effect of metal film for light, and the total energy reflectance has a strong narrow band reflection effect. This feature is designed for the security of our narrowband optical zoom high-reflection film is meaningful.

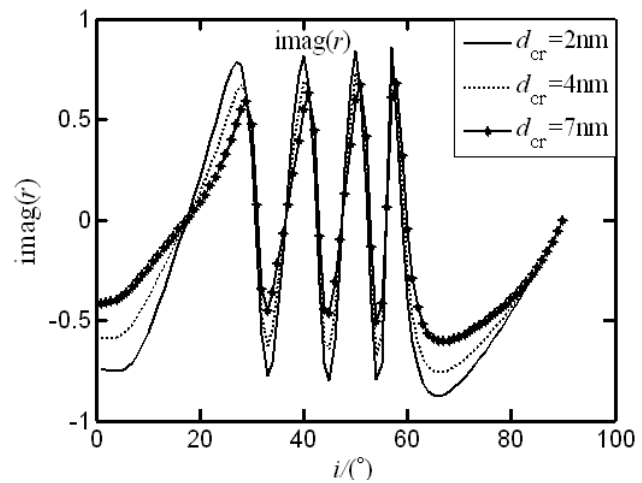

Fig.3 The reflection coefficient (imaginary part)of light of multilayer film

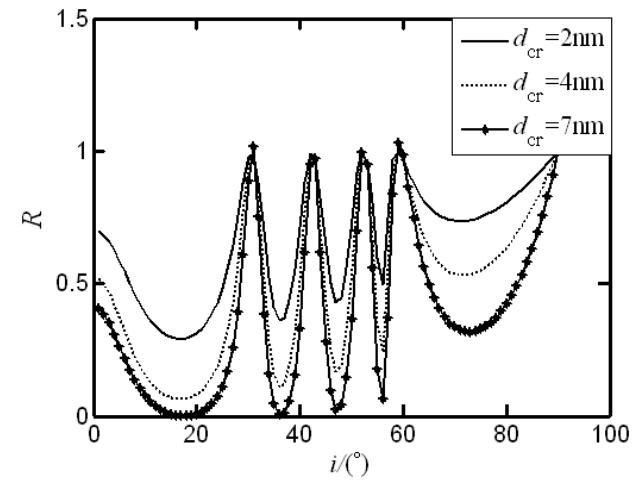

Fig.4 The energy reflectivity of multilayer film

\section{Conclusion}

With the boundary conditions of inhomogeneous electromagnetic waves, the relationship equation is derived for the derived S- polarized light waves propagates in multilayer (containing thin metal film) membrane system. Ultra-thin metal film with a simplified processing, realized the 
computation of energy reflectivity for combination discoloration security film system containing thin metal film, through practical examples of the algorithm to achieve the study, the calculation results of numerical examples show that the effect of chromium film is displayed, but the change of the total energy reflectivity is small with the thickness of chromium film increasing, a narrow band reflection effect exhibits stronger. These features for us to design the narrowband high reflected anti-counterfeiting optical film will have important reference value.

\section{Acknowledgements}

This work was Supported by Supported by the Beijing City Board of Education Science and technology key project (KZ20150015015), Supported by Beijing Natural Science Foundation (4142016), Supported by the Beijing City Board of Education Science and technology project(KM201510015009), Supported by BIGC Key Project(E-a-2013-19).

\section{References}

[1] Zhang J F, Liu L M,Ye Y D. Optical anti-counterfeiting technology and its application(in Chinese). Beijing: National Defense Industry Press,2011: 175-216

[2] Zhang Y X, Chen J Shi G C. Color Prediction Model of Optically Variable Anti-forgery Ink. Packaging Engineering,2011,32(5): : 94-96

[3] Tan M Q, Lin Y C, Zhao D Z. The properties of periodic symmetrical coatings and the Design of high reflectivity coatings of narrow band for containing the ultra-thin metallic film( $(\mathrm{n}=\mathrm{k})$ (in Chinese). Acta Photonica Sinica, 1996,25(1):1011-1017.

[4] Mathieu H , Hersch R D. Classical print reflection models: A radiometric approach [ J ] . Imaging S ci T echno ,2004, 48( 4) : 363--373.

[5] Xu J H. The Electromagnetic Field and Electromagnetic Wave in Layer Media (in Chinese). Beijing: Petroleum Industry Press, 1997: 38-59

[6] Liu F P, Wang A L, Chen Q,et al. The research progress on lateral shift of reflected electromagnetic wave at the interface of two conductive media. Chinese Science Bulletin, 2008,53:961-968

[7] Wang A L,Liu FP,Zhu X F, et al.The Reflection Properties of Multilayer Anti-counterfeiting Optical films for Vertical-polarized wave Acta Photonica Sinica, 2014,43(8): 0831002-1 6.

[8] Xu Z Y. The printability of optical falsification-resistant ink color prediction model of optically variable ink [Master degree thesis]. Jiang Nan university, 2011:19-35 